\title{
Management of inadvertent template contamination in production of oligonucleotide qPCR reagents
}

\author{
Ivan Sadowski*,1,3 iD \& Aaron Bogutz ${ }^{2,3}$ \\ ${ }^{1}$ Department of Biochemistry \& Molecular Biology, LSI University of British Columbia Vancouver, BC, Canada; ${ }^{2}$ Department of Medical Genetics, LSI University of British \\ Columbia Vancouver, BC, V6T 1Z3, Canada; ${ }^{3}$ Department of Molecular Epigenetics, LSI University of British Columbia Vancouver, BC, V6T 1Z3, Canada; ${ }^{\star}$ Author for \\ correspondence: ijs.ubc@gmail.com
}

BioTechniques 69: 401-403 (December 2021) 10.2144/btn-2020-0129

First draft submitted: 24 August 2020; Accepted for publication: 21 September 2020; Published online: 24 November 2020

\section{KEYWORDS:}

diagnostics $\bullet$ oligonucleotide primers $\bullet$ qPCR $\bullet$ remedial action $\bullet$ template contamination

The quantitative polymerase chain reaction, qPCR, is an important routine tool for diagnosis of microbial infections [1]. This assay employs forward and reverse oligonucleotide primers for exponential amplification of pathogen-specific nucleic acids by thermostable DNA polymerases. Amplification of target sequences can be detected in each cycle by fluorescence produced by double stranded DNAspecific fluorescent dyes, or release of fluorescent dyes coupled to quenched oligonucleotide pathogen-specific probes [2]. Double stranded DNA-specific dyes (e.g., SYBR, EVA Green) provide a sensitive and inexpensive option for monitoring amplification of specific sequences but are usually limited to detection of a single target sequence per reaction. In contrast, fluorescently labeled 'TaqMan ${ }^{\circledR}$ ' oligonucleotide probes allow for 'multiplexing', enabling the detection of multiple specific sequences from samples in a single reaction [3]. This capability is particularly important for clinical diagnosis, which may require internal positive controls or detection of multiple specific nucleic acid sequences within thousands or more routine samples [3]. During the current COVID-19 pandemic, caused by the coronavirus SARS-CoV-2, diagnostic labs around the world are utilizing this technology to identify infections [4].

Sensitive diagnostic detection of pathogens from clinical samples using qRT-PCR can detect as little as ten or fewer copies of template DNA or RNA [5]. Consequently, these assays require that all reagents - including oligonucleotides, fluorescent probes, enzymes and nucleotides - be free of contamination by pathogen-specific nucleic acids. As production of detection reagents and control templates has scaled up during the COVID-19 pandemic, there have been multiple reports of reagents from commercial suppliers of oligonucleotide probes and primers that produced amplification in nontemplate (NT)/nonsample control reactions, indicating background contamination by viral sequences (pers. comm.). These inadvertent contaminations may have been introduced through manipulation of reagents by asymptomatic infected individuals, or more likely by close proximity to laboratory production of SARS-CoV-2 nucleic acids.

During the present COVID-19 pandemic, many research groups have redirected some focus toward assisting with diagnosis, or the development of therapies or vaccines. To support local diagnostic efforts, we organized a collaboration to produce test reagents, including enzymes, oligonucleotides and TaqMan probes specific for SARS-CoV-2. For production of oligonucleotides and probes, we used an ABI394 synthesizer, on which we produced primers for amplification of nucleic acids specific for the SARS-CoV-2 RNA-dependent RNA polymerase $(R d R P)$ and $E$ genes to detect the virus, in addition to the human $R N A s e P$ positive control [4,5]. Initial oligonucleotides from our instrument produced results for detection of viral sequences comparable to those obtained with oligos purchased from commercial suppliers.

To enable routine quality control and analysis of our test reagents, we synthesized positive control template nucleic acids for the $R d R P$ and $E$ gene target sequences, which were cloned into pSP72. Both template fragments were synthesized as six overlapping 30 to 60-nt oligonucleotides on the same ABI394 instrument as was used for production of the diagnostic oligos. The full approximately 200-nucleotide fragments were produced using a single round of assembly PCR, digested with restriction enzymes for sites included at the $5^{\prime}$ and $3^{\prime}$ ends and cloned into pSP72. Clones of these constructs were grown in miniprep cultures, and DNA was prepared and analyzed in rooms adjacent to open space housing the DNA synthesizer.

Unexpectedly, the oligo primer sets synthesized immediately following construction and growth of the template plasmids failed quality control, in that they produced amplification with a cycle threshold (Ct) value of approximately 31 in no-template (NT) controls, indicating background template contamination; we confirmed this result ourselves for both the RdRP and $E$ gene primers with our own enzymes (Figure 1A and not shown, respectively). This observation was surprising because, although the same synthesizer was used to produce the PCR primers and template oligos, no other shared reagents or equipment were used for downstream processing of the oligonucleotides and production of the cloned plasmid templates. The only common parameters were the individual operating the 


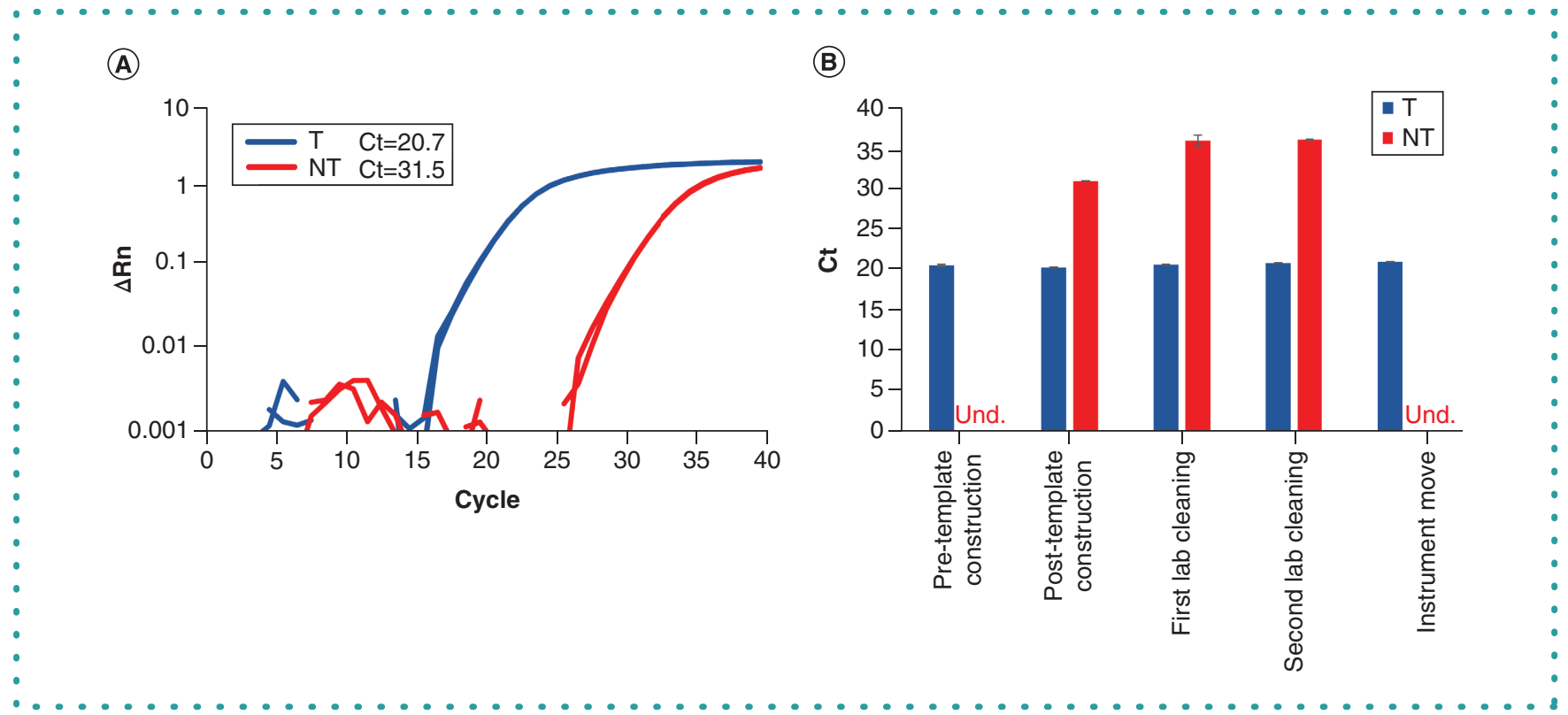

Figure 1. qPCR detection of contaminating nucleic acids. (A) Amplification plot of T and NT reactions immediately following production of template plasmids. (B) T and NT Ct values for each step of cleaning performed. Error bars indicate standard deviation. Ct: Cycle threshold; NT: Nontemplate; T: Template; Und.: Undetermined.

synthesizer and the open proximity of the synthesizer, SpeedVac and heat block used for oligo processing to the laboratory space where the control template plasmids were produced and expanded as minipreps.

In response, we thoroughly cleaned every available instrument and bench surface near the ABI394, and any surface in the adjacent space that had been used for production of the template clones, with a $10 \%$ bleach solution. We also purged the immediately adjacent laboratory of any cloned template samples or PCR products, and all subsequent work with these was performed on a separate floor of our building. Following this initial remedial action, we produced primers for RdRP; these were assayed immediately, but we again observed amplification in the NT control reaction with a Ct of 36 (Figure 1B, first lab cleaning). This indicates that our cleaning efforts had reduced the source of template contamination by a factor of roughly $32\left(2^{5}\right)$, but despite this improvement these reagents would not pass quality control by our associated clinical diagnostic laboratory. We performed a more thorough round of surface cleaning, whereby we also eliminated any reagents used for oligo processing (including acetonitrile, $\mathrm{dH}_{2} \mathrm{O}$ and $\mathrm{NH}_{4} \mathrm{OH}$ ) that were present in the lab during construction of the templates. These measures had no appreciable effect; primers produced immediately afterward also produced amplification in the NT control with a Ct of 36 (Figure 1B, second lab cleaning). We then realized that more drastic measures would be required if we were to resume production of diagnostic oligos.

Because we did not have access to another synthesizer, we decided to relocate our instrument to a space sufficiently removed from our laboratory, and in this process avoid carryover of template contamination. Consequently, we moved the instrument to an adjacent corridor from which all reagent bottles were removed, in addition to the delivery and pressure lines for the acetonitrile and dichloromethane reservoirs (bottles 18 and 19). We cleaned all exterior surfaces of the instrument thoroughly with DNA AWAY ${ }^{\top M}$ solution (Thermo Fisher, MA, USA) prior to moving it to space in a separate wing of our building. We did not have a spare argon pressure line or regulator, so these were cleaned in a similar way. The instrument was reassembled with new bottles at all positions, new acetonitrile vials, new pressure/delivery lines to bottles $18 / 19$ and a new argon tank. The precolumn ferrules were also replaced for each of the four column mounts. Each delivery line was purged with a minimum of $100 \mathrm{ml}$ acetonitrile for bottles 9-19 or $20 \mathrm{ml}$ for reagent vials 1-5. The instrument was then prepared for synthesis by installing fresh bottles of all reagents, and a new set of RdRP-specific primers were synthesized. All downstream oligo processing steps, including deprotection, desalting and drying, were performed with a different heat block and SpeedVac, located in an adjacent space to the relocated synthesizer. Additional measures that were implemented included ensuring that nitrile gloves were worn at all times during operation of the instrument and processing of the oligonucleotides, and that none of the reagents or consumables had been exposed to our original lab space. Suspensions of the newly produced oligos were also manipulated using micropipettes borrowed from another laboratory. Importantly, the first RdRP-specific oligonucleotide set synthesized on the relocated instrument produced amplification from the control template but no detectable amplification in a control reaction without template (Figure 1B, instrument move). This result was confirmed by quality control analysis of these oligos provided to a diagnostic lab. Subsequently, we have produced multiple synthesis of the SARS-CoV-2 RdRP and E gene-specific diagnostic primers, all of which 
have passed quality control analysis for background template contamination. These results indicate that our drastic measures were successful in eliminating template contamination from our oligo production process.

Our experience is not unique; two local diagnostic laboratories have received oligonucleotides from several suppliers that did not pass NT quality control (pers. comm.). Furthermore, we note that at least one commercial supplier currently requires an indication as to whether requested oligos target SARS-CoV-2 sequences, such that manufacture can be performed to 'minimize risks of contamination by viral templates'. This study highlights the ease with which template nucleic acids can contaminate PCR reagents, particularly oligonucleotides, and the extreme measures needed to remedy this; however, prevention is the most potent tool against potential contamination. Segregation of template production from synthesis of PCR reagents is highly advised, ideally on different machines in physically separate rooms. Importantly, we demonstrate that it is possible to rescue use of an oligonucleotide synthesizer that had been exposed to template contamination. Following our remediation, no further contaminated reagents were produced. The current COVID-19 pandemic will persist for at least another year (and likely much longer); consequently, it is imperative to maintain and improve testing capacity. Furthermore, because qPCR is required for diagnosis of many common seasonal infections, the availability of high-quality reagents, free of template contamination, will be required until an alternative technology is developed to detect infections.

\section{Author contributions}

I Sadowski performed oligonucleotide synthesis, instrument and lab cleaning and wrote the manuscript; A Bogutz performed qPCR analysis, wrote the manuscript and prepared the figures.

\section{Financial \& competing interests disclosure}

This research was supported by funds provided by Genome British Columbia and the Canadian Institutes of Health Research. The authors have no other relevant affiliations or financial involvement with any organization or entity with a financial interest in or financial conflict with the subject matter or materials discussed in the manuscript apart from those disclosed.

No writing assistance was utilized in the production of this manuscript.

\section{Open access}

This work is licensed under the Attribution-NonCommercial-NoDerivatives 4.0 Unported License. To view a copy of this license, visit http://creativecommons.org/licenses/by-nc-nd/4.0/

\section{References}

1. Evans MF. The polymerase chain reaction and pathology practice. Diagn. Histopathol. (Oxf.) 15(7), 344-356 (2009).

2. Navarro E, Serrano-Heras G, Castaño MJ, Solera J. Real-time PCR detection chemistry. Clin. Chim. Acta 439, 231-250 (2015).

3. Lute S, Wang H, Sanchez D, Barletta J, Chen Q, Brorson K. Multiplex RT qPCR assay for simultaneous quantification of three viruses used for validation of virus clearance by biopharmaceutical production. Biologicals 37(5), 331-337 (2009).

4. Tang Y-W, Schmitz JE, Persing DH, Stratton CW. Laboratory diagnosis of COVID-19: current issues and challenges. J. Clin. Microbiol. 58(6), e00512-20 (2020).

5. Hirotsu Y, Mochizuki H, Omata M. Double-quencher probes improve detection sensitivity toward Severe Acute Respiratory Syndrome Coronavirus 2 (SARS-CoV-2) in a reverse-transcription polymerase chain reaction (RT-PCR) assay. J. Virol. Methods 284, 113926 (2020). 
